\title{
Weakest Bus Identification Based on Modal Analysis and Singular Value Decomposition Techniques
}

\author{
M. K. Jalboub, H. S. Rajamani, R.A. Abd-Alhameed and A. M. Ihbal \\ School of engineering, Design and Technology University of Bradford, Bradford, BD7 1DP, UK \\ $\{$ m.k.jalboub, h.s.rajamani , r.a.a.abd, a.m.i.ihbal\}@ Bradford.ac.uk
}

\begin{abstract}
Voltage instability problems in power system is an important issue that should be taken into consideration during the planning and operation stages of modern power system networks. The system operators always need to know when and where the voltage stability problem can occur in order to apply suitable action to avoid unexpected results. In this paper, a study has been conducted to identify the weakest bus in the power system based on multi-variable control, modal analysis, and Singular Value Decomposition (SVD) techniques for both static and dynamic voltage stability analysis. A typical IEEE 3machine, 9-bus test power system is used to validate these techniques, for which the test results are presented and discussed.
\end{abstract}

Keywords: Voltage stability, multi-variable control, modal analysis, singular value decomposition, power system dynamics.

\section{INTRODUCTION}

During the last decades, many of researches have been conducted in the area of voltage stability problems and their relation to the power system operation and control. These researches deal with the voltage stability problem as static and static analysis methods are used based on the study of the reduced $(V-Q)$ Jacobian matrix, and by applying modal analysis [1-6]. As a result, the participation factors of bus, branch and generator on the static voltage stability can be obtained. In addition, the margin to voltage stability limit and the minimum distance to instability can also be obtained [6]. However, power system network is consists of a large number of dynamic system and its dynamic behavior has great influence on the voltage stability. Recently, the most blackouts have shown that voltage stability problems is associated with issues of frequency and angle stability $[7,8]$. Consequently, it is necessary to take the full dynamic system model of the power network into consideration in order to get more precise results. A few researchers have been conducted on the dynamic voltage stability analysis [1, $5,6,9]$. The common structure of the system model used is analogous to that used for transient stability analysis. The overall structure of the system equations of dynamic system under analysis consists of a set of first-order differential equations in addition to the algebraic equations (DAEs) [6]. On the other hand, for voltage stability study, particular interest should be given to issues of reactive power and voltage control and load actions. By minimizing oscillations of the state and network variables the function of dynamic voltage stability analysis is achieved [9]. Followed by, a parameter optimization method is applied for limiting the magnitude of oscillations. The voltage stability is decoupled from the angle dynamics as stated in [10]. The researchers are assuming that all electromechanical oscillations are considered to be stable, by neglecting the power-angle dynamics of the power system, the voltage response of the uncontrolled power system can be calculated by using the eigenvalue of the Voltage Stability Matrix.

Nevertheless, in bulky power system networks, the dynamic voltage stability is coupled with different modes of oscillations such as exciter mode, local mode and inter-area mode of oscillations. While there is many study have been conducted on voltage stability problems, only some of them deal with this matter as dynamic.

In this paper, an approach for the evaluation of dynamic voltage stability is proposed. The way takes the advantages of modern multi-variable control theory. Based on the Multi-Input Multi-Output (MIMO) transfer function, interactions between suitably defined input and output variables affecting dynamic voltage stability can be analyzed at different frequency modes.

\section{2.1 SMALL SIGNAL VOLTAGE STABILITY ANALYSIS}

The mathematical model for the dynamic voltage stability study of a power system comprises a set of first order differential equations and a set of algebraic equations $[1,6]$ : $\dot{x}=f(x, y)$

$0=g(x, y)$

Where $x$ is the state vector of the system and $y$ vector containing bus voltages

The numerical integration and power flow analysis methods can be used to solve equations (1\&2) in the time domain by $[5,6]$. To evaluate the steady state equilibrium values $\left(x_{0}, y_{0}\right)$ of the dynamic system, the derivatives in equation (1) equal is 
set equals to zero. Through the linearization about $\left(x_{0}, y_{0}\right)$, equation $(1 \& 2)$ are expressed as follows $[1,5]$ :

$$
\frac{d \Delta x}{d t}=A \Delta x+B \Delta y
$$

$0=c \Delta x+D \Delta y$

Further, by eliminating $\Delta y$, the linearised state equation can be rewritten as $[1,5]$ :

$$
\frac{d \Delta x}{d t}=\left(A-B D^{-1} C\right) \Delta x=\tilde{A} \Delta x
$$

The static bifurcation will occur when $\operatorname{det}(D)=0$. In order to investigate the dynamic bifurcation occurrence, it is always assumed that $\operatorname{det}(D) \neq 0$ and that matrix inverse is exists $[1$, 5]. By analyzing the eigenvalue of $\tilde{A}$, dynamic voltage stability can be performed.

Static Voltage Stability Analysis Based on Reduced Jacobian Matrix

The static voltage stability analysis is based on the modal analysis of the power flow of the Jacobian matrix given by [16]:

$$
\left[\begin{array}{c}
\Delta P_{p Q, P V} \\
\Delta Q_{P Q}
\end{array}\right]=\left[\begin{array}{ll}
J_{p \theta} & J_{P V} \\
J_{Q \theta} & J_{Q V}
\end{array}\right] \cdot\left[\begin{array}{c}
\Delta \theta \\
\Delta V_{P Q}
\end{array}\right]
$$

Where

$\triangle P_{P Q, P V}:$ The incremental change in bus real power.

$\Delta Q_{P Q} \quad$ : The incremental change in bus reactive power.

$\Delta \theta \quad:$ The incremental change in bus voltage angle.

$\Delta V \quad$ : The incremental change in bus voltage magnitude.

The elements of the Jacobian matrix represent the sensitivity between nodal power and bus voltage changes [11]. On the other hand, the phenomenon of power system voltage stability is mostly affected by the reactive power variation. By keeping real power invariable at each operating point, the $Q-V$ analysis can be performed. Assuming $\triangle P_{P Q, P V}=0$, it follows from equation (6) [11, 12]:

$$
\Delta Q_{P Q}=\left(J_{Q V}-J_{Q \theta} * J_{P \theta}^{-1} * J_{P V}\right) * \Delta V_{P Q}=J_{R} * \Delta V_{P Q}
$$

And:

$$
\Delta V_{P Q}=J_{R}^{-1} * \Delta Q_{P Q}
$$

Based on the $J_{R}^{-1}$, which is the reduced $V-Q$ Jacobian matrix, the $Q-V$ modal analysis can be performed. Consequently, the participation factors of bus, branch and generator are calculated. Moreover, the stability margin and the shortest distance to instability of power system under investigation will be determined [11-12].

The application of singular value decomposition to the reduced Jacobian matrix $J_{R}^{-1}$ also allows the static voltage stability analysis $[13,14,15]$.

\section{Small signal stability analysis based on Multi-Variable control technique}

By a multivariable technique we mean a process with several inputs and several outputs as shown in Fig. (1). In general, every input is connected to every output through some dynamic coupling. We can pretend that the $i^{\text {th }}$ output $y_{i}$ is connected to the $j^{\text {th }}$ input $u_{i}$ through a transfer function $g_{i j}(s)$, we can write:

$$
y_{i}(s)=\sum_{j=1}^{r} g_{i j}(s) * U_{i}(s)
$$

Or

$$
\left[\begin{array}{c}
y_{i}(s) \\
\vdots \\
y_{m}(s)
\end{array}\right]=g_{i j}(s)\left[\begin{array}{c}
u_{i}(s) \\
\vdots \\
u_{r}(s)
\end{array}\right]
$$

Where the notation $g_{i j}(s)$ indicates the transfer function matrix, that is:

$$
g_{i j}(s)=\left[\begin{array}{c}
g_{i j}(s) \cdots g_{i r}(s) \\
\vdots \\
g_{m j}(s) \cdots g_{m r}(s)
\end{array}\right]
$$

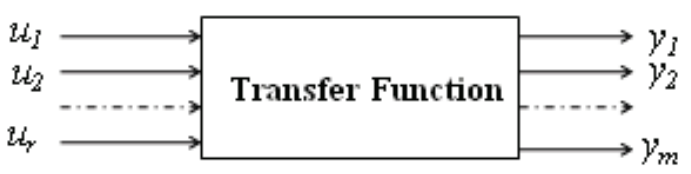

Figure 1 A multivariable technique: a system with $r$ inputs and $m$ outputs

In this paper, voltage dynamic stability analysis based on the Multi-Input Multi-Output (MIMO) transfer technique is carried out. Fig. (2) Shows MIMO system which is widely used in control engineering. The requirements for this analysis detailed dynamic power system model including generators, governors, static exciter, power system stabilizer, and non-linear voltage and frequency load models is necessary. Furthermore, dynamic load models may also included. In general, the dynamic models described by equation (1\&2) must consider all relaxant issues affecting voltage stability.

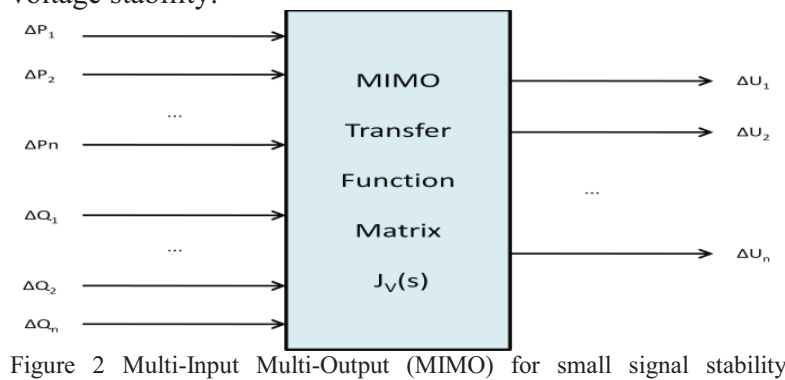

Figure 2 Multi-Input Multi-Output (MIMO) for small signal stability modeling 


\section{Multi-Input Multi-output Techniques (MIMO model)}

As the first step, variables that affecting dynamic voltage stability must be selected as input variables to the MIMO system. These variables are the real and reactive power control functions of chosen generators and loads. Some other variables, such as the tap-changer position and FACTS control signals, can also be included as inputs. The output signals for a MIMO are the voltage magnitudes at the most critical buses. In this paper, the generation and load controls parameters were used as the input signals to a MIMO system transfer function matrix. Moreover, due to the small size of the simulated power system model, the set of output signals are extended to all bus voltages. The transfer function that corresponding to MIMO model shown in Fig. (2) is described by equation (13):

$\left[\begin{array}{c}\Delta U_{1} \\ \Delta U_{2} \\ \vdots \\ \Delta U_{n}\end{array}\right]=J_{v}(s)\left[\begin{array}{c}\Delta P_{1} \vdots \Delta Q_{1} \\ \Delta P_{2} \vdots \Delta Q_{2} \\ \vdots \\ \Delta P_{n}: \Delta Q_{n}\end{array}\right]$

Where

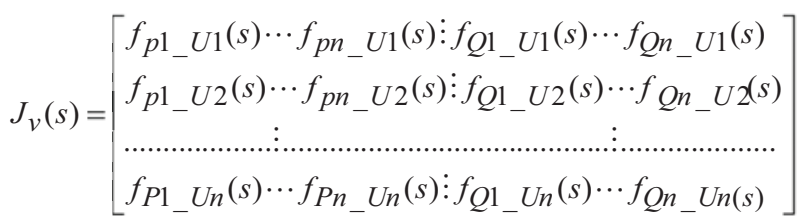

$J_{\nu}(s)$ is a $n_{\text {output }} * n_{\text {input }}$ transfer function matrix.

To obtain each sub-transfer function in the $J_{v}(s)$ a numerical methods can be applied to power system dynamic model.

Small signal stability analysis based on singular value decomposition (SVD) Techniques

In order to analyze the MIMO system, SVD of $J_{v}(s)$ is carried out at every fixed frequency $[14,16,18]$ :

$J_{v}(s)=U S V^{T}$

Where $S$ is $n_{\text {output }} * n_{\text {input }}$ matrix with $\mathrm{k}=\min \{$ $\left.n_{\text {output }} * n_{\text {input }}\right\}$ non-negative singular values, $\sigma_{i}$ are arranged in descending order along its main diagonal; the other entire's are zero. The singular values are the positive square roots of the eigenvalue of $J_{v}^{T}(S) * J_{v}(S)$, where $J_{v}^{T}(S)$ is the complex conjugate transpose of $J_{v}(S)$ [19]:

$\sigma_{i}\left(J_{v}(S)\right)=\sqrt{\lambda_{i}\left(J_{v}^{T}(S) * J_{v}(S)\right)}$

$\mathrm{U}$ is $n_{\text {output }} * n_{\text {input }}$ unitary matrix of output singular vectors, $u_{i}$

$\mathrm{V}$ is $n_{\text {input }} * n_{\text {input }}$ unitary matrix of input singular vectors, $v_{i}$.

Singular vectors analysis

The column vectors of $U$, denoted $u_{i}$, represent the directions of the output variables. They are orthogonal and of unit length. Likewise, the column vectors of $V$, denoted $v_{i}$, represent the directions of input variables. These input and output variables are connected through the singular values [16].

In the case of dynamic stability, the singular values and their associated directions vary with the frequency. Critical frequencies corresponding to poorly damped dominant modes must be taken into account in power system dynamic voltage stability analysis studies.

To obtain the relationship between input and output, the maximum singular values and their associated related input and output singular vectors are analyzed at each frequency. The output singular vector shows at which bus voltage magnitude is the most critical bus. The input singular vectors indicate which input has the greatest influence on the corresponding output. As a result, by means of the singular value analysis technique, the dynamic voltage stability of the power system can be conducted.

\section{SIMULATION RESULTS}

\section{Test system description}

A typical IEEE 3- machine, 9-bus test power system model $[6,17]$, as shown in Fig (3). is used for demonstration of the proposed MIMO system. The corresponding power system dynamic model consists of a set 3 generators each described by $6^{\text {th }}$ order model, governors, static exciters, Power System Stabilizer (PSS) , 9 load buses and 6 branches (transmission lines). The detailed generator, controller, branches and load model can be found in $[6,20]$.

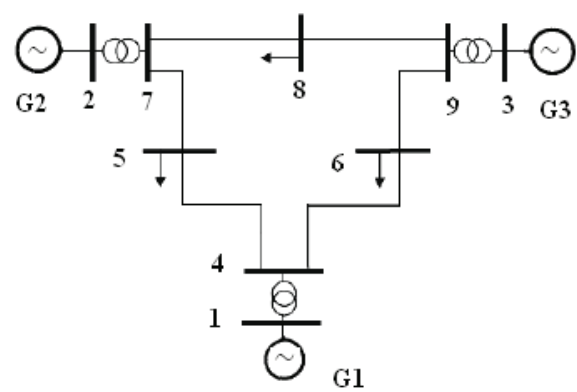

Figure 3 IEEE 3-machines, 9-bus test system

\section{Experiments}

To assess the dynamic voltage stability of the proposed test power system the flowing steps is performed:

- Static voltage stability analysis based on singular value decomposition to observe the selected nodes and suitable control input variable for detailed 
dynamic voltage stability analysis.

- Modal analysis to find the critical modes of oscillations; the frequency range of critical modes is known, so that the detailed modal analysis can be omitted.

- Transfer function matrix $J_{v}(s)$ calculation and SVD at critical frequencies.

- Find the critical buses, which have the most severe dynamic voltage stability problem

- Find the best input variables allowing improvement of voltage dynamic stability.

Weak bus identification by based on singular value decomposition (Static voltage stability)

Since the reduced Jacobian matrix $J_{R}^{-1}$, as given in equation (8), is a square matrix, therefore, both singular value analysis and modal analysis can be applied as a static indicator for voltage stability and weak bus identification. Based on the theory given in $[13,14,15]$, the static voltage stability is analyzed based on the singular value technique in this paper.

Applying the singular value analysis to IEEE 3- machine, 9bus test power system model, it found that the maximum singular value of the reduced Jacobian matrix $J_{R}^{-1}$ is 0.16 and the corresponding maximum output singular vector is 0.68 and it associates with bus 8 . This means the turbulence on the reactive loads power will cause the largest variation on the voltage magnitude at bus 8 . As a result, bus 8 is ranked as the weakest bus in the test system. The simulation result is shown in Fig. (4).

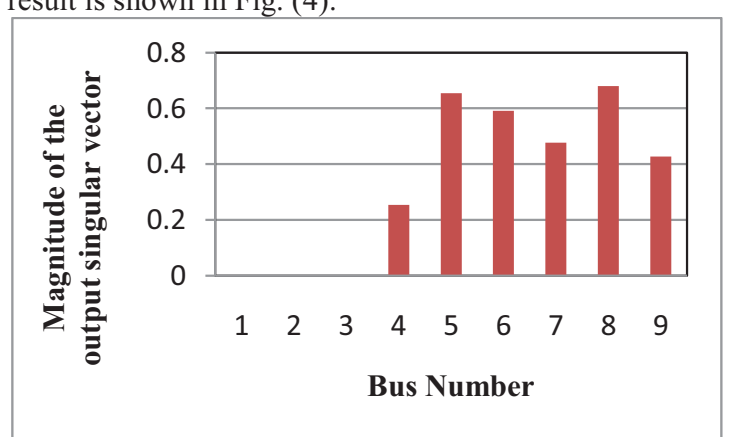

Figure 4 Plot of magnitude of the output singular vector vs bus number

\section{IV.WEAK BUS IDENTIFICATION BASED ON MODAL ANALYSIS AND SINGULAR VALUE TECHNIQUE}

\section{Modal analysis technique}

Modal analysis is performed to find the critical modes of oscillations. It found that the exciter mode has a damping ratio of $11.93 \%$ and the oscillation frequency is about 2.82 $\mathrm{Hz}$. The inter-area mode has a damping ratio of $13.63 \%$ and the corresponding oscillation frequency is about $0.57 \mathrm{~Hz}$.

\section{Singular value technique}

Detailed dynamic power system model is used to calculate the transfer function matrix $J_{v}(s)$ based on the numerical method. The maximum singular value of $J_{v}(s)$ provides the maximal gain between the input and output variables. It describes how the observed outputs can be influenced by the inputs [21]. The maximal singular value of the reduced Jacobian matrix $J_{R}^{-1}$ over the frequency range of $[0.01 \mathrm{~Hz}$ to about $100 \mathrm{~Hz}$ ] is carried out. As a result, the peaks of the maximal singular value that correspond to the inter-area mode and exciter mode 1 were established respectively.

According to the previous analysis, the singular value is performed for the two critical oscillation mode frequencies i.e. exciter mode and inter-area mode.

\section{Exciter Mode}

The results of singular value analysis shows that the output singular vectors that corresponding to the maximum singular value at the exciter mode frequency is given in Fig. (5). It can be seen that the buses 5,6 , and 8 are related to the dynamic voltage stability with bus 8 standing out as the most critical bus. The analysis of the input singular vector associated with this mode shows that the input signals of $\mathrm{Q}_{2}, \mathrm{Q}_{3}$, and $\mathrm{Q}_{1}$ (input number 16,17 and 18) are the most suitable signals for this mode of dynamic voltage stability control. The other input signals have relative weak influences as can been seen in Fig. (6).

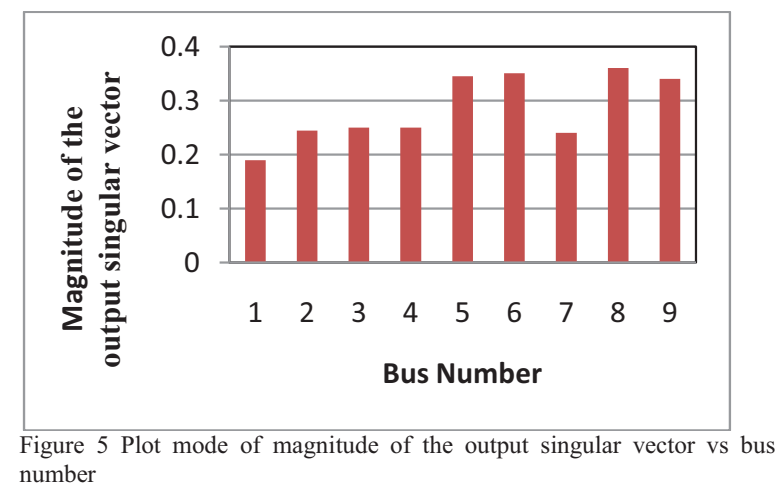

\section{Inter-area Mode}

The critical output singular vector associated with the interarea mode oscillation mode is shown in Fig. (7). From the figure, it is clear that the bus 8 is the most critical bus. In comparison with exciter mode 1 , the inter-area mode has larger output singular vectors.

Fig. (8) Shows the analysis of the input singular vector associated with inter-area mode. It is clear that the input signal of $\mathrm{Q}_{2}, \mathrm{Q}_{3}$ and $\mathrm{Q}_{1}$ are the most suitable signals for dynamic stability analysis and control (input number 16, 17 and 18) 


\section{CONCLUSIONS}

This paper presents the application of multi-variable control (MIMO), modal analysis, and singular value decomposition to study the static and dynamic voltage stability in order to identify the weakest bus in the power system. The singular value technique was to analyze the MIMO.

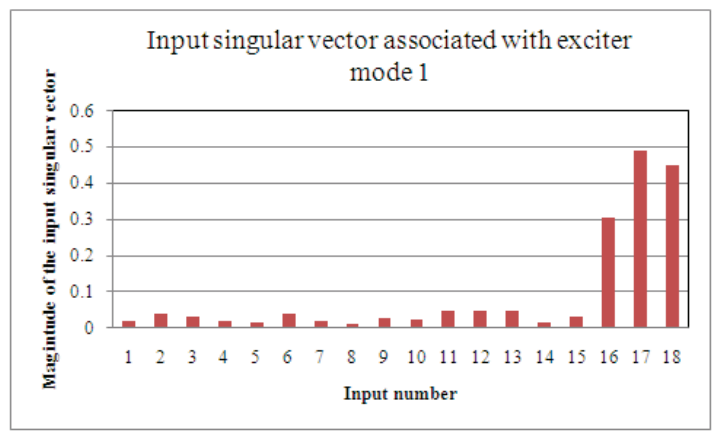

Figure 6 Plot of input singular vector associated with exciter mode 1

The elements of the input singular vectors indicate the impact of the input variables on the output variables, and the output singular vectors can be used to evaluate the influence of voltage stability on the selected buses. Moreover, the input singular vector can be used for choosing the most suitable control action to improve voltage stability, and the output singular vector can provide information about the weakest buses that affected by voltage stability. A typical IEEE 3machine, 9-bus test power system is used to validate these techniques and the results were presented.

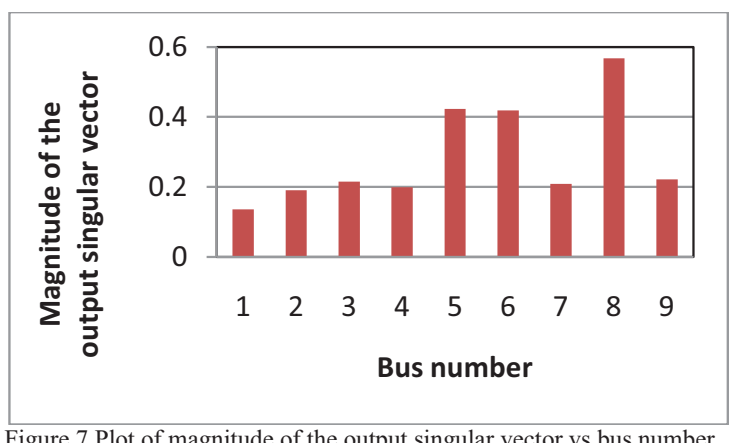

\section{REFERENCES}

[1] IEEE Power Engineering Society/ CIGRE, FACTS Overview, Publication 95TP108, IEEE Press, New York, 1995.

[2] N. G. Hingorani and L. Gyugyi, Understanding FACTS, IEEE Press, New York, 1999.

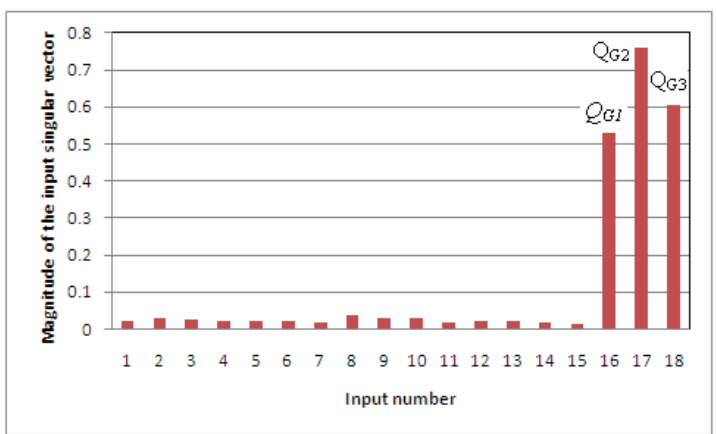

Figure 8 Plot of magnitude input singular vector vs input number

[3] Qiu Xiaoyan; Li Xingyuan; Xu Jian; Xia Lili; , "AC/DC Hybrid Transmission System Voltage Stability Analysis Based on Singular Value Decomposition Method," Power and Energy Engineering Conference (APPEEC), 2010 Asia-Pacific , vol., no., pp.1-4, 28-31 March 2010.

[4] Avalos, R.J.; Canizares, C.A.; Anjos, M.F.; , "A practical voltagestability-constrained optimal power flow," Power and Energy Society General Meeting - Conversion and Delivery of Electrical Energy in the 21st Century, 2008 IEEE, vol., no., pp.1-6, 20-24 July 2008.

[5] W. H. Litzenberger, Ed., "An Annotated Bibliography of High-Voltage Direct-Current Transmission, 1989-1991," Published by the Bonneville Power Administration (BPA) and the Western Area Power Administration, Portland, OR, 1992.

[6] W. H. Litzenberger, Ed., "An Annotated Bibliography of High-Voltage Direct-Current Transmission and Flexible AC Transmission (FACTS) Devices, 1991-1993," Published by the Bonneville Power Administration (BPA) and the Western Area Power Administration, Portland, OR, 1994.

[7] W. H. Litzenberger and R. K. Varma, Eds., "An Annotated Bibliography of High- Voltage Direct-Current Transmission and FACTS Devices, 1994-1995," Published by the Bonneville Power Administration (BPA) and the U.S. Department of Energy, Portland, OR, 1996.

[8] W. H. Litzenberger, R. K. Varma, and J. D. Flanagan, Eds., “An Annotated Bibliography of High-Voltage Direct-Current Transmission and FACTS Devices, 1996-1997," Published by the Electric Power Research Institute (EPRI) and the Bonneville Power Administration (BPA), Portland, OR, 1998.

[9] CIGRE Task Force 14-27, "Unified Power Flow Controller," CIGRE Technical Brochure, 1998.

[10] L. Gyugyi, "A Unified Power Flow Control Concept for Flexible AC Transmission Systems,” IEE Proceedings-C, Vol. 139, No. 4, July 1992.

[11] B. A. Renz et al., "AEP Unified Power Flow Controller Performance," IEEE Transactions on Power Delivery, Paper 1998. [40] B. A. Renz et al., "World's First Unified Power Flow Controller on the AEP System," CIGRE Paper No. 14-107, 1998. 
[12] K. K. Sen and E. J. Stacey, "UPFC-Unified Power Flow Controller: Theory, Modelling, and Applications,” Presented at the IEEE/ PES 1998 Winter Meeting, New York.

[13] R. Mihalic, P. Zunko, and D. Povh, "Improvement of Transient Stability Using Unified Power Flow Controller," IEEE Transactions on Power Delivery, Vol. 11, No. 1, 1996, pp. 485-491.

[14] J. Y. Liu and Y. H. Song, "Comparison Studies of Unified Power Flow Controller With Static Var Compensators and Phase Shifters," Electric Machines and Power Systems, Vol. 27, No. 3, 1999.

[15] M. Noorozian, L. Angquist, M. Ghandhari, and G. Andersson, "Use of UPFC for Optimal Power Flow Control," IEEE Transactions on Power Delivery, Vol. 12,No. 4, 1997, pp. 1629-1634.

[16] S. Arabi and P. Kundur, "A Versatile FACTS Device Model for Power Flow and Stability Simulations," IEEE Transactions on Power Systems, Vol. 11, No. 4, November 1996, pp. 1944-1950.

[17] A. Nabavi-Niaki and M. R. Iravani, "Steady-State and Dynamic Models of Unified Power Flow Controller (UPFC) for Power System Studies," IEEE Transactions on Power Systems, Vol. 4, 1996, pp. 1937-1943.

[18] C. D. Schauder, L. Gyugyi, M. R. Lund, D. M. Hamai, T. R. Reitman, D. R. Torgerson, and A. Edris, "Operation of the Unified Power Flow Controller (UPFC) Under Practical Constraints," IEEE Transactions on Power Delivery, Vol. 13, No. 2, April 1998.

[19] G. Rogers, " Power System Oscillations", Kluwer Academic Publishers, Dec. 1999.

[20] S. Skogestad and I. Potlethwaite, "Multivariable feedback controlAnalysis and design”, John Wiley \&Sons, July 1996.

[21] M. Rahman et al., "UPFC Application on the AEP System: Planning Considerations," IEEE Transactions on Power Systems, Vol. 12, No. 4, November 1997. 